\title{
Les bienfaits pour la santé et la prédominance du sucre dans les céréales pour déjeuner destinées aux enfants au Canada
}

\author{
Monique Potvin Kent, Ph. D. (1); Cher Cameron, B. Sc. (2); Sarah Philippe, B. Sc. (2)
}

Cet article a fait l'objet d'une évaluation par les pairs.

Diffuser cet article sur Twitter

\section{Résumé}

Introduction : Cette étude vise à comparer le contenu nutritionnel et les bienfaits pour la santé des céréales pour déjeuner destinées aux enfants et celles non destinées aux enfants et à évaluer la prédominance du sucre ajouté dans ces produits.

\begin{abstract}
Méthodologie : Nous avons recueilli des données sur le contenu nutritionnel de 262 céréales pour déjeuner vendues dans les cinq principales chaînes d'alimentation à Ottawa (Ontario) et à Gatineau (Québec). Pour chaque céréale, nous avons pris en note les cinq premiers ingrédients et la quantité de sucres ajoutés indiqués sur la liste des ingrédients. Les diverses marques de céréales ont été ensuite classées en deux catégories, soit "plus saines » ou « moins saines », à l'aide du modèle de profil nutritionnel du Royaume-Uni. Nous avons évalué chaque céréale en fonction de divers critères afin de déterminer si elle était destinée ou non aux enfants. Des comparaisons statistiques ont été établies entre les céréales destinées aux enfants et les autres.
\end{abstract}

Résultats : Sur l'ensemble des céréales pour déjeuner, 19,8 \% étaient destinées aux enfants et contenaient beaucoup moins de gras et de gras saturés. Ces céréales avaient une teneur en sodium et en sucre significativement plus élevée et une teneur en fibres et en protéines plus faible, et elles étaient trois fois plus susceptibles d'être classées comme « moins saines » par rapport aux céréales non destinées aux enfants. Aucune des céréales destinées aux enfants n'était sans sucre et, pour $75 \%$ d'entre elles, le sucre occupait le deuxième rang dans la liste des ingrédients. Six entreprises de céréales pour déjeuner possédaient une gamme de produits destinés aux enfants composée entièrement de céréales « moins saines ».

Conclusion : Il est nécessaire d'adopter un règlement qui limite le marketing alimentaire ciblant les enfants et les jeunes de moins de 17 ans sur l'emballage, en vue de réduire l'attrait envers ce groupe d'âge. Il faut également modifier la composition des céréales pour déjeuner visant les enfants au moyen de cibles établies par le gouvernement ou par la réglementation si on juge sa conformité inacceptable.

Mots-clés : déjeuner, enfants, nutrition, céréale, obésité, approvisionnement alimentaire, sucre, marketing

\section{Introduction}

Le monde industrialisé a connu une hausse substantielle du surpoids et de l'obésité chez les enfants, les taux ayant doublé entre 1980 et 2008'. Cette situation a entraîné un risque accru de maladies cardiovasculaires, de diabète, de troubles musculosquelettiques et de divers cancers à l'échelle mondiale ${ }^{2}$. Au Canada, le taux d'obésité juvénile a augmenté de manière spectaculaire au cours en 30 ans, passant de $2 \%$ en 1981 à $12 \%$ en 2009-2011, et le taux combiné de surpoids et d'obésité chez les enfants de 5 à 17 ans s'élève actuellement à $31,5 \%{ }^{3,4}$.

Le marketing des aliments et des boissons a été associé à l'obésité juvénile, de même que les préférences alimentaires des enfants,

\section{Points saillants}

- Comparativement aux autres céréales, les céréales destinées aux enfants :

- avaient une teneur en fibres, en protéines, en gras totaux et en gras saturés significativement plus faible; et

- avaient une teneur en sodium et en sucre significativement plus élevée.

- Les céréales destinées aux enfants étaient trois fois plus susceptibles d'être classées " moins saines » que les autres céréales.

- Parmi les céréales destinées aux enfants, aucune n'était sans sucre, et la majorité contenait deux ou trois types de sucres ajoutés.

- Six entreprises de céréales pour déjeuner possédaient une gamme de produits destinés aux enfants composée entièrement de céréales « moins saines».

la consommation à court terme d'aliments et les demandes alimentaires ${ }^{5,6}$. La recherche a montré que la majorité des produits faisant l'objet de publicités ciblant les enfants et les jeunes sont riches en matières grasses, en sucre et en sodium et comportent une faible valeur nutritive ${ }^{7-9}$. L'Organisation mondiale de la santé (OMS) recommande que les pays limitent le nombre de publicités d'aliments et de boissons vues par les enfants dans toutes les formes de médias et dans les lieux où les enfants se réunissent ${ }^{10}$. La première recommandation comprend l'étiquetage des emballages. $\mathrm{Au}$ Canada, c'est l'industrie qui règlemente en grande partie le marketing des aliments et des boissons par l'intermédiaire de l'Initiative canadienne pour la publicité sur les aliments et boissons destinés aux 
enfants ${ }^{11}$. Cette initiative a été lancée en 2007 par 16 entreprises d'aliments et de boissons qui se sont engagées à annoncer seulement des produits sains ou à cesser de faire de la publicité visant les enfants de moins de 12 ans à la télévision, à la radio, ainsi que dans les médias imprimés et numériques (comme Internet et les téléphones intelligents). Aucun engagement n'a été pris relativement à l'emballage ou à l'étiquetage des produits alimentaires. Au Québec, toute publicité à des fins commerciales destinée aux enfants de moins de 13 ans est interdite en vertu de la Loi sur la protection du consommateur, qui a été mise en œuvre en 1980 dans le but de protéger les enfants de la publicité en général ${ }^{12}$. Cette loi interdit la publicité destinée aux enfants dans presque tous les types de médias, dont la télévision et Internet, dans les écoles et les garderies. L'étiquetage est toutefois exclu, ce qui fait qu'aucune province ni aucun territoire au Canada ne protège les enfants contre la publicité figurant sur les emballages de produits.

Les céréales pour déjeuner représentent une catégorie de produits fortement commercialisée auprès des enfants. Aux ÉtatsUnis, des études ont révélé qu'en moyenne, en 2015, les enfants de 2 à 11 ans avaient vu plus de 500 publicités de céréales pour déjeuner rien qu'à la télévision ${ }^{13}$ et, en 2009, les publicités de céréales visant les enfants occupaient le deuxième rang en matière de dépenses en publicité, juste derrière la restauration rapide ${ }^{14}$. Au Canada, les enfants de 2 à 11 ans voient en moyenne 33 publicités de céréales pour déjeuner par mois rien qu'à la télévision ${ }^{15}$. Les céréales pour déjeuner sont la troisième catégorie de produits les plus fréquemment annoncés, représentant $11 \%$ de la publicité, tant à la télévision destinée aux enfants que sur leurs sites Internet préférés ${ }^{16,17}$.

Aux États-Unis, la recherche a montré que le contenu nutritionnel des céréales pour déjeuner destinées aux enfants est extrêmement faible ${ }^{18-19}$. Selon une analyse de tous les aliments ciblant les enfants et vendus dans les supermarchés canadiens en 2008, dans $93 \%$ des céréales pour déjeuner, plus de $20 \%$ des calories provenaient du sucre ${ }^{20}$. Une méta-analyse a permis d'établir qu'une consommation élevée de sucre chez les enfants (particulièrement les boissons sucrées) est associée à un risque accru d'obésité ${ }^{21}$. Ces résultats préoccupent les responsables de la santé publique.
Aucune étude canadienne ne s'est attardée spécifiquement au contenu nutritionnel global des céréales pour déjeuner visant les enfants ni n’a comparé les céréales destinées aux enfants aux autres céréales. Compte tenu de la forte prévalence du marketing des céréales pour déjeuner ciblant les enfants, l'objectif principal de notre recherche était de comparer le contenu nutritionnel et les bienfaits pour la santé des céréales pour déjeuner destinées aux enfants et des autres céréales pour déjeuner. L’objectif secondaire était de vérifier la prédominance en sucres ajoutés dans les céréales à déjeuner pour enfants au Canada. Cet objectif revêt une importance particulière, car Santé Canada a récemment passé en revue la réglementation relative à l'étiquetage des aliments et proposé que les sucres soient regroupés dans la liste des ingrédients afin de permettre aux consommateurs de repérer plus facilement les sources de sucre dans les produits alimentaires $^{22}$. Notre hypothèse était que les céréales pour déjeuner destinées aux enfants étaient moins saines que les autres céréales pour déjeuner, et qu'elles contenaient une plus grande quantité de sucre. Enfin, le troisième objectif de notre étude était de déterminer quelles entreprises devraient améliorer les bienfaits pour la santé de leurs céréales pour enfants.

\section{Méthodologie}

Nous avons conçu une étude transversale afin d'évaluer le contenu nutritionnel et les bienfaits pour la santé des céréales destinées aux enfants et des autres céréales.

\section{Collecte des données nutritionnelles}

Trois adjoints de recherche (en quatrième année d'études de premier cycle) ont constitué un échantillon de commodité à Ottawa (Ontario) et à Gatineau (Québec) visant les cinq principaux détaillants en alimentation au Canada selon les ventes ${ }^{23}$, à savoir Loblaws, Sobeys (qui appartient à Empire Co.), Métro, Costco et Wal-Mart, et ils ont compilé une liste de toutes les céréales pour déjeuner prêtes à manger offertes sur le marché. Ils ont retiré les produits en double et consigné le nom des entreprises. Un adjoint de recherche s'est ensuite rendu dans les commerces en question et a pris des photos de tous les côtés de chacune des boîtes de céréales inscrites sur la liste. L'information nutritionnelle de chaque céréale (sans lait ajouté) provient ainsi directement du tableau des valeurs nutritives figurant sur la boîte. Elle concerne la portion (g), le nombre total de calories (cal), les gras trans (g), les gras saturés (g), le sodium (mg), les fibres (g), les sucres (g) et les protéines (g). À l'exception des gras trans, la collecte de ces nutriments était nécessaire pour la répartition des aliments dans les deux catégories de céréales, " plus saines " ou " moins saines ». Nous avons recueilli l'information sur les gras trans car il est prouvé qu'ils sont particulièrement néfastes pour la santé, étant donné qu'ils augmentent le taux sérique de lipoprotéines de faible densité (LDL) et réduisent le taux sérique de lipoprotéines de haute densité (HDL) ${ }^{24}$.

Pour chaque céréale, nous avons noté la quantité de sucres ajoutés à partir de la liste d'ingrédients et pris en note leur ordre sur celle-ci. La réglementation de Santé Canada précise en effet que les ingrédients figurant sur les produits alimentaires préemballés doivent être énumérés dans l'ordre décroissant de leur poids ${ }^{25}$. Les sucres ajoutés étaient l'agave, la cassonade, le sucre de canne ou le jus de sucre de canne évaporé, les jus de fruits concentrés, le sirop de maïs, le dextrose ou la dextrine, le fructose, le galactose, le glucose, le glucose-fructose, le sirop de maïs à haute teneur en fructose, le miel, le sucre inverti, le sucre liquide, le maltose, le sirop d'érable, la mélasse, le nectar, le sucre brut, le sucrose, le sirop et le sucre blanc. Nous avons ensuite pris en note les cinq premiers ingrédients présentés dans la liste d'ingrédients des céréales dans l'ordre de leur apparition.

\section{Classification nutritionnelle}

Les aliments ont été répartis dans les catégories de céréales "plus saines » ou « moins saines » à l'aide du modèle de profil nutritionnel en trois étapes du RoyaumeUni, élaboré par la Food Standards Agency du Royaume-Uni ${ }^{26}$. Ce modèle a été choisi parce que sa validité et sa fiabilité ont été jugées bonnes ${ }^{27,28}$ et qu'il a été utilisé de façon efficace dans diverses études de recherche en vue de déterminer avec exactitude la qualité nutritionnelle des aliments $^{7,29}$. Pour réaliser cette classification, chaque nutriment a été converti par portion de $100 \mathrm{~g}$ de céréales et des points ont été attribués en fonction de la quantité d'énergie $(\mathrm{kJ})$, de gras saturés $(\mathrm{g})$, de sucres totaux (g), de protéines $(\mathrm{g})$, de fibres (g), de sodium (mg) et en fonction du pourcentage de fruits, de légumes et de noix, selon les tableaux fournis par un 
rapport d'orientation sur le modèle de profil nutritionnel du Royaume-Uni ${ }^{26}$. Nous avons ensuite effectué les calculs suivants pour chaque céréale : (points attribués à l'énergie + points attribués aux gras saturés + points attribués aux sucres + points attribués au sodium) - (points attribués au pourcentage de fruits, de légumes et de noix + points attribués aux fibres + points attribués aux protéines). Un produit ayant obtenu moins de 4 points était considéré comme " plus sain » et un produit ayant obtenu 4 points ou plus était considéré comme $"$ moins sain $»^{26}$.

\section{Évaluation du ciblage des enfants}

L'expression "destiné aux enfants » que nous avons utilisée repose sur des recherches antérieures portant sur la publicité d'aliments et de boissons ciblant les enfants sur l'emballage et à la télévi$\operatorname{sion}^{20,30}$. Une céréale pour déjeuner a été considérée comme destinée directement aux enfants si elle contenait des friandises, des images destinées aux enfants (p. ex. bande dessinée) ou des messages conçus pour attirer leur attention (p. ex. "Hé, les enfants! »), si elle encourageait leur interaction avec le produit (p. ex. casse-tête ou jeu) ou si son nom de marque ou son logo faisait mention d'enfants, si elle faisait un lien avec des émissions de télévision pour enfants, des films ou des prestations musicales ou si elle utilisait des couleurs primaires et des polices de style bande dessinée pour attirer les enfants. Si aucun des éléments susmentionnés ne s'appliquait, la céréale faisait alors partie de la catégorie " non destinée aux enfants ». Pour réaliser cette classification, deux des adjoints de recherche ont examiné les six côtés des boîtes de céréales et tout désaccord a été réglé par le chercheur principal. La fiabilité interévaluateurs, qui était de $95 \%$, a été calculée comme suit : 1 - (12 désaccords / 262 boîtes de céréales) $\times 100$. L'ensemble des activités de collecte de données a été effectué à l'automne 2015.

\section{Analyse statistique}

Nous avons réalisé les analyses statistiques à l'aide du logiciel IBM SPSS Statistics, version 23.0 (IBM Corp., Armonk, New York, États-Unis). Une analyse de la moyenne $(\bar{x})$ et de l'écart-type (ET) de chaque nutriment a été effectuée pour l'échantillon total, puis pour les céréales destinées aux enfants et pour les autres céréales. Les tests $t$ ont permis de déterminer si les différences étaient statistiquement significatives. Nous avons calculé le nombre de céréales pour déjeuner " plus saines » et " moins saines », puis calculé le chi carré $\left(\chi^{2}\right)$ et le rapport de cotes afin d'évaluer les différences entre les céréales destinées aux enfants et les autres céréales. Par la suite, nous avons déterminé le nombre de céréales destinées aux enfants et de céréales non destinées aux enfants "plus saines » et " moins saines" par entreprise. Les entreprises produisant un petit nombre de produits à base de céréales (p. ex. moins de cinq produits) ont été regroupées sous la catégorie « autres entreprises ». Dorset Cereal, Small Planet Foods, A\&V 2000 Inc., Fourmi Bionique, naturSource, Empire Company Limited, Food for Life, GoGo Quinoa, Swissli, Wal-Mart et WildRoots faisaient partie de cette catégorie. Enfin, la quantité de sucre par céréale et l'ordre des ingrédients ont été présentés sous forme de tableau, et des statistiques descriptives ont servi à calculer la proportion d'aliments contenant des sucres ajoutés et le nombre de fois où les sucres ajoutés apparaissaient dans la liste d'ingrédients.

\section{Résultats}

Nous avons repéré au total 266 céréales pour déjeuner, mais, lors de visites subséquentes dans les supermarchés, quatre céréales ont été impossibles à trouver parce qu'elles n'étaient plus offertes sur le marché, ce qui fait que nous avons analysé 262 céréales. De ce nombre, 52 (19,8 \%) étaient destinées aux enfants. En moyenne, les céréales destinées aux enfants contenaient des quantités significativement plus élevées de sodium (439,7 mg en moyenne contre $266,2 \mathrm{mg}$ pour les autres céréales) et de sucre (30,2 g en moyenne contre 19,2 g pour les autres céréales) (tableau 1). Les céréales destinées aux enfants avaient également une teneur significativement plus faible en fibres (5,2 $\mathrm{g}$ en moyenne contre 9,6 g pour les autres céréales) et en protéines (6,3 g en moyenne contre 10,0 g pour les autres céréales). Par contre, les céréales destinées aux enfants avaient une teneur significativement plus faible en gras totaux $(3,6$ g en moyenne contre 7,2 g pour les autres céréales) et en gras saturés $(0,7 \mathrm{~g}$ en moyenne contre $1,5 \mathrm{~g}$ pour les autres céréales). La majorité des céréales, destinées ou non aux enfants, ont été classées comme "moins saines " sur la base du modèle de profil nutritionnel du RoyaumeUni (tableau 2) et nous avons observé une association significative entre les bienfaits pour la santé et le ciblage des enfants $\left(\chi^{2}=7,6[d l=1], p=0,006\right)$ : les céréales

TABLEAU 1

Quantité moyenne de nutriments par 100 g de céréales pour déjeuner destinées aux enfants et de céréales pour déjeuner non destinées aux enfants, Ottawa et Gatineau, Canada

\begin{tabular}{|c|c|c|c|c|c|}
\hline & $\begin{array}{c}\text { Toutes les céréales } \\
\qquad \overline{\mathbf{x}},(\mathrm{ET})\end{array}$ & $\begin{array}{c}\text { Céréales destinées } \\
\text { aux enfants } \\
\overline{\mathbf{x}},(\mathrm{ET})\end{array}$ & $\begin{array}{c}\text { Céréales non destinées } \\
\text { aux enfants } \\
\overline{\mathbf{x}},(\mathrm{ET})\end{array}$ & Test $t$ (dl) & Valeurs $p$ \\
\hline Gras totaux (g) & $6,5(5,8)$ & $3,6(3,4)$ & $7,2(6,1)$ & $5,593(140,0)$ & 0,001 \\
\hline Gras saturés (g) & $1,4(2,0)$ & $0,7(1,5)$ & $1,5(2,0)$ & $3,475(102,3)$ & 0,001 \\
\hline Sodium (mg) & $300,7(219,3)$ & $439,7(217,2)$ & $266,3(206,2)$ & $-5,370(260,0)$ & 0,001 \\
\hline Fibres $(\mathrm{g})$ & $8,7(5,7)$ & $5,2(3,4)$ & $9,6(5,9)$ & $5,175(260,0)$ & 0,001 \\
\hline Sucres (g) & $21,4(10,5)$ & $30,2(11,6)$ & $19,2(9,1)$ & $-6,396(67,3)$ & 0,001 \\
\hline Protéines (g) & $9,3(3,7)$ & $6,3(2,0)$ & $10,1(3,7)$ & $9,911(146,8)$ & 0,001 \\
\hline
\end{tabular}

Abréviations : $d l$, degrés de liberté; ET, écart-type; $\bar{x}$, moyenne. 
TABLEAU 2

Nombre et pourcentage de céréales pour déjeuner destinées aux enfants et de céréales pour déjeuner non destinées aux enfants classées comme " plus saines » et « moins saines » d'après le modèle de profil nutritionnel du Royaume-Uni

\begin{tabular}{lccccc}
$\begin{array}{l}\text { Qualité des } \\
\text { céréales }\end{array}$ & $\begin{array}{c}\text { Toutes les } \\
\text { céréales } \\
\mathbf{n}(\%)\end{array}$ & $\begin{array}{c}\text { Destinées } \\
\text { aux enfants }\end{array}$ & $\begin{array}{c}\text { Non } \\
\text { destinées aux } \\
\text { enfants }\end{array}$ & $\chi^{2}(\boldsymbol{d})$ & Valeur $\boldsymbol{p}$ \\
\hline «Plus saines » & $82(31,3)$ & $8(15,4)$ & $74(35,2)$ & & \\
«Moins saines » & $180(68,7)$ & $44(84,6)$ & $136(64,8)$ & $7,6(1)$ & 0,006 \\
Total & $262(100,0)$ & $52(100,0)$ & $210(100,0)$ & & \\
\hline
\end{tabular}

Abréviation : $d l$, degrés de liberté.

destinées aux enfants étaient trois fois plus susceptibles d'être classées dans la catégorie « moins saines » que les autres céréales.

Dans l'ensemble, seulement $7,3 \%$ ( $n=19$ ) des céréales pour déjeuner étaient sans sucre, et beaucoup contenaient deux ou trois types de sucre ( $\mathrm{n}=127 ; 48,5 \%$ ), comme l'illustre le tableau 3. Aucune céréale destinée aux enfants n'était sans sucre et la majorité ( $\mathrm{n}=31 ; 59,6 \%$ ) contenait deux ou trois types de sucres ajoutés. Au total, $9 \%(n=19)$ des céréales non destinées aux enfants étaient sans sucre et $45,7 \%(\mathrm{n}=96)$ contenaient deux ou trois sucres ajoutés. Presque $6 \%$ de ces céréales $(\mathrm{n}=12)$ contenaient entre sept et onze types de sucre.

En général, le premier ingrédient le plus courant dans les céréales était l'avoine $(38,9 \%)$, le sucre étant le deuxième ou troisième ingrédient le plus fréquent (dans respectivement $44,3 \%$ et $35,5 \%$ des cas), comme l'illustre le tableau 4. Le premier ingrédient le plus présent dans les céréales destinées aux enfants était le maïs (30,8 \%), suivi du blé entier $(26,9 \%)$ et de l'avoine $(19,2 \%)$, le sucre arrivant au deuxième

TABLEAU 3

Nombre de sucres présents dans les céréales pour déjeuner destinées aux enfants et dans les céréales pour déjeuner non destinées aux enfants, Ottawa et Gatineau, Canada

\begin{tabular}{lrrrrrr} 
Nombre de sucres & $\begin{array}{c}\text { Toutes les céréales } \\
\text { n (\%) }\end{array}$ & \multicolumn{2}{c}{$\begin{array}{c}\text { Céréales destinées aux } \\
\text { enfants } \\
\text { n (\%) }\end{array}$} & \multicolumn{2}{c}{$\begin{array}{c}\text { Céréales non destinées } \\
\text { aux enfants } \\
\mathbf{n}(\%)\end{array}$} \\
\hline Aucun & 19 & $(7,3)$ & 0 & $(0,0)$ & 19 & $(9,0)$ \\
1 & 47 & $(17,9)$ & 9 & $(17,3)$ & 38 & $(18,1)$ \\
2 à 3 & 127 & $(48,5)$ & 31 & $(59,6)$ & 96 & $(45,7)$ \\
4 à 6 & 56 & $(21,4)$ & 11 & $(21,2)$ & 45 & $(21,4)$ \\
7 à 11 & 13 & $(5,0)$ & 1 & $(1,9)$ & 12 & $(5,7)$ \\
Total & 262 & $(100,0)$ & 52 & $(100,0)$ & 210 & $(100,0)$ \\
\hline
\end{tabular}

par General Mills, Métro, Nature's Path Foods, Post, Quaker et Sally's faisait partie de la catégorie des céréales « moins saines».

\section{Analyse}

Cette étude a permis de constater que $85 \%$ des céréales pour déjeuner destinées aux enfants vendues dans la région d'OttawaGatineau étaient « moins saines » d'après le modèle de profil nutritionnel du RoyaumeUni, et que ces céréales étaient trois fois plus susceptibles que les autres céréales d'être classées comme " moins saines ". Dans notre échantillon, General Mills avait le plus grand nombre de céréales destinées aux enfants $(n=14)$ et la totalité de ses céréales étaient classées " moins saines ". Kellogg's arrivait au deuxième rang quant au nombre de céréales destinées aux enfants ( $\mathrm{n}=16$ ), avec $63 \%$ classées « moins saines ». Dans le cas des entreprises comme Métro $(n=6)$, Nature's Path Foods $(n=5)$, Post $(n=3)$ et Sally's $(n=3)$, la totalité des céréales destinées aux enfants étaient " moins saines ». Malgré le fait que 10 céréales destinées aux enfants appartiennent à la catégorie " moins saines », Kellogg's s'est démarquée en étant l'entreprise qui offrait le plus grand nombre de céréales destinées aux enfants «plus saines » $(n=6)$. La gamme de céréales " plus saines » produites par les entreprises de céréales pour déjeuner doit de toute évidence être étendue, d'autant plus que les céréales pour déjeuner font l'objet d'une promotion intense auprès des enfants dans d'autres médias $^{16,30}$

Autre constatation importante : les céréales destinées aux enfants contenaient, en moyenne, des quantités significativement plus élevées de sucre et de sodium et des quantités plus faibles de fibres et de protéines par rapport aux autres céréales. La forte teneur en sucre dans les céréales pour déjeuner destinées aux enfants - $30 \mathrm{~g}$ sur $100 \mathrm{~g}$ de céréales en moyenne (ou $31 \%$ de l'apport énergétique) - est inquiétante, étant donné que les recherches ont montré que la consommation de sucre, surtout de sucres ajoutés, est directement liée à l'obésitée ${ }^{1}$. Notre étude a également permis de démontrer qu'aucune céréale destinée aux enfants n'était sans sucre, contre $9 \%$ des céréales non destinées aux enfants. La majorité $(60 \%)$ des céréales destinées aux enfants contenait deux ou trois types de sucres ajoutés, $23 \%$ de ces céréales contenant au moins quatre types de sucres ajoutés et, pour $75 \%$ d'entre elles, le sucre était le deuxième ingrédient en importance. 


\section{TABLEAU 4}

Les trois ingrédients les plus courants dans les céréales pour déjeuner destinées aux enfants et les céréales pour déjeuner non destinées aux enfants, Ottawa et Gatineau, Canada

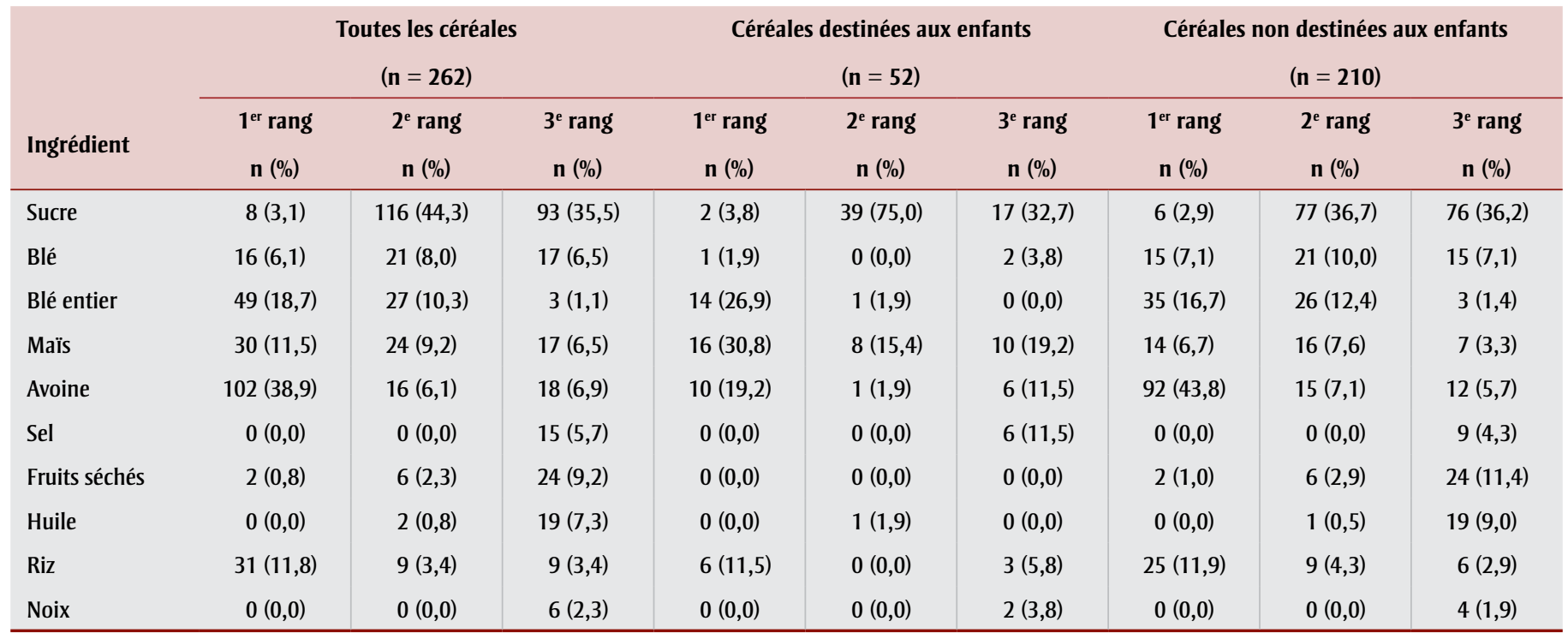

D’autres travaux de recherche menés au Canada ont révélé que les sucres libres (c.-à-d. les sucres ajoutés et les sucres d'origine naturelle dans les jus de fruits) sont présents dans $64 \%$ de l'ensemble des produits emballés au pays ${ }^{31}$. L'OMS recommande de ramener l'apport individuel en sucres libres à $10 \%$ ou moins de l'apport énergétique total ${ }^{32}$. D’après nos résultats, la consommation de sucre d'un enfant sédentaire de 8 ans, dont l'apport calorique devrait être de 1500 calories $^{33}$ et qui consomme $50 \mathrm{~g}$ de céréales destinées aux enfants par jour, serait d'environ $15 \mathrm{~g}$ (ou $60 \mathrm{kcal}$ ) en moyenne, ce qui représente $40 \%$ de son apport total en sucres libres pour la journée. Comme les céréales pour déjeuner ne constituent qu'une source de sucres ajoutés parmi d'autres dans l'alimentation des enfants et que, aux États-Unis, ces produits se classent au sixième rang des sources de sucre pour les enfants de 2 à 18 ans après les boissons sucrées, les desserts à base de céréales, les boissons aux fruits, les desserts à base de produits laitiers et les friandises ${ }^{34}$, ces enfants consomment probablement beaucoup plus que la limite de sucre de $10 \%$ recommandée par l'OMS. Il faut donc réduire la teneur en sucre des céréales destinées aux enfants, ce qui pourrait être réalisé au départ par des cibles fédérales imposées aux aliments transformés, puis par l'imposition d'un règlement si on estime que l'industrie se conforme insuffisamment à ces cibles. C'est cette approche qui est adoptée au Royaume-Uni, où la Public Health England a demandé à l'industrie de réduire la quantité de sucre dans les produits fréquemment consommés par les enfants d'au moins $20 \%$ d'ici 2020. Si les objectifs ne sont pas atteints, le gouvernement envisagera l'adoption d'un règlement officiel ${ }^{35}$. La réduction du sucre dans les céréales destinées aux adultes est également recommandée, car même si ces céréales contiennent une quantité moyenne de sucre significativement moins élevée par 100 g de céréales que les céréales destinées aux enfants, dans notre échantillon, un nombre important (46\%) renfermait deux ou trois sucres ajoutés par céréale, et $27 \%$ en contenaient au moins quatre types.

En 2015, au moment où nos données ont été collectées, la politique d'étiquetage relative au sucre permettait aux fabricants de produits alimentaires d'indiquer sur l'étiquette les différents types de sucre en ordre décroissant de poids. Les fabricants pouvaient ainsi éviter d'afficher le sucre comme premier ingrédient de la liste dans un produit alimentaire en ajoutant plusieurs types de sucres et en les énumérant séparément. Santé Canada a récemment mis à jour les dispositions de la Loi sur les aliments et drogues concernant l'étiquetage des aliments : les sucres doivent dorénavant être regroupés dans la liste des ingrédients afin de permettre aux consommateurs de repérer plus facilement les sources de sucre dans les produits alimentaires ${ }^{22}$. Les fabricants ont jusqu'en décembre 2021 pour regrouper tous les sucres en un ingrédient, par exemple sous la forme : «Ingrédients : sucres (sucre, sirop de maïs, fructose) ». Cette politique pourrait conduire les fabricants de céréales à réduire la quantité de sucres dans leurs produits, puisqu'ils voudront probablement éviter d'afficher le sucre en premier sur la liste des ingrédients.

Nous avons également constaté que les céréales destinées aux enfants avaient une teneur significativement élevée en sodium : elles contenaient en moyenne $440 \mathrm{mg}$ de sodium par $100 \mathrm{~g}$, contre $226 \mathrm{mg}$ pour les autres céréales. Les recherches montrent que, chez les enfants et les adolescents, la pression artérielle systolique et le risque d'hypertension s'accentuent au même rythme que leur consommation de sodium $^{36,37}$. L'OMS recommande de réduire l'apport individuel en sodium à $2 \mathrm{~g}$ par jour pour les adultes, et moins pour les enfants, en fonction de leurs besoins énergétiques ${ }^{38}$.

Les céréales pour déjeuner destinées aux enfants avaient également une teneur en fibres beaucoup plus faible. Elles contenaient en moyenne $5 \mathrm{~g}$ de fibres par $100 \mathrm{~g}$, contre $9 \mathrm{~g}$ par $100 \mathrm{~g}$ pour les autres céréales. Cela signifie que, pour une portion de $50 \mathrm{~g}$ de ce type de céréales, un enfant ne consommerait que $2,6 \mathrm{~g}$ de fibres en moyenne - environ $10 \%$ seulement de l'apport suffisant en fibres totales recommandé, lequel varie de 25 à $31 \mathrm{~g}$ par jour selon l'âge et le sexe pour les enfants de 4 à 13 ans $^{39}$. Malgré certains résultats contradictoires ayant trait au lien particulier entre un apport accru en fibres alimentaires chez 
TABLEAU 5

Nombre et pourcentage de céréales pour déjeuner destinées aux enfants et de céréales pour déjeuner non destinées aux enfants classées comme " plus saines » et « moins saines », par entreprise, Ottawa et Gatineau, Canada

\begin{tabular}{|c|c|c|c|}
\hline Nom de l'entreprise & $\begin{array}{c}\text { Toutes les céréales } \\
\text { n (\%) }\end{array}$ & $\begin{array}{c}\text { « Plus saines » } \\
\text { n (\%) }\end{array}$ & $\begin{array}{c}\text { « Moins saines » } \\
\text { n (\%) }\end{array}$ \\
\hline General Mills & $35(100,0)$ & $4(11,4)$ & $31(88,6)$ \\
\hline Destinées aux enfants & $14(100,0)$ & - & $14(100,0)$ \\
\hline Non destinées aux enfants & $21(100,0)$ & $4(19,0)$ & $17(81,0)$ \\
\hline Jordan's (Grain Product Limited) & $10(100,0)$ & - & $10(100,0)$ \\
\hline Destinées aux enfants & - & - & - \\
\hline Non destinées aux enfants & $10(100,0)$ & - & $10(100,0)$ \\
\hline Kellogg's & $48(100,0)$ & $17(35,4)$ & $31(64,6)$ \\
\hline Destinées aux enfants & $16(100,0)$ & $6(37,5)$ & $10(62,5)$ \\
\hline Non destinées aux enfants & $32(100,0)$ & $11(34,4)$ & $21(65,6)$ \\
\hline Love Grown Foods & $9(100,0)$ & $2(22,2)$ & $7(77,8)$ \\
\hline Destinées aux enfants & - & - & - \\
\hline Non destinées aux enfants & $9(100,0)$ & $2(22,2)$ & $7(77,8)$ \\
\hline Métro & $14(100,0)$ & $6(42,9)$ & $8(57,1)$ \\
\hline Destinées aux enfants & $6(100,0)$ & - & $6(100,0)$ \\
\hline Non destinées aux enfants & $8(100,0)$ & $6(75,0)$ & $2(25,0)$ \\
\hline Nature's Path Foods & $45(100,0)$ & $16(35,6)$ & $29(64,5)$ \\
\hline Destinées aux enfants & $5(100,0)$ & - & $5(100,0)$ \\
\hline Non destinées aux enfants & $40(100,0)$ & $16(40,0)$ & $24(60,0)$ \\
\hline Post & $14(100,0)$ & $5(35,7)$ & $9(64,3)$ \\
\hline Destinées aux enfants & $3(100,0)$ & - & $3(100,0)$ \\
\hline Non destinées aux enfants & $11(100,0)$ & $5(45,5)$ & $6(54,5)$ \\
\hline Choix du président & $32(100,0)$ & $13(40,6)$ & $19(59,4)$ \\
\hline Destinées aux enfants & - & - & - \\
\hline Non destinées aux enfants & $32(100,0)$ & $13(40,6)$ & $19(59,4)$ \\
\hline Quaker (Pepsi) & $10(100,0)$ & $2(20,0)$ & $8(80,0)$ \\
\hline Destinées aux enfants & $1(100,0)$ & - & $1(100,0)$ \\
\hline Non destinées aux enfants & $9(100,0)$ & $2(22,2)$ & $7(77,8)$ \\
\hline Sally’s (MOM Brands Company) & $9(100,0)$ & $1(11,1)$ & $8(88,9)$ \\
\hline Destinées aux enfants & $3(100,0)$ & - & $3(100,0)$ \\
\hline Non destinées aux enfants & $6(100,0)$ & $1(16,7)$ & $5(83,3)$ \\
\hline Weetabix & $7(100,0)$ & $6(85,7)$ & $1(14,3)$ \\
\hline Destinées aux enfants & $2(100,0)$ & $1(50,0)$ & $1(50,0)$ \\
\hline Non destinées aux enfants & $5(100,0)$ & $5(100,0)$ & - \\
\hline Autres entreprises & $29(100,0)$ & $10(34,5)$ & $19(65,5)$ \\
\hline Destinées aux enfants & $2(100,0)$ & $1(50,0)$ & $1(50,0)$ \\
\hline Non destinées aux enfants & $27(100,0)$ & $9(33,3)$ & $18(66,7)$ \\
\hline Total (\%) & $262(100,0)$ & $82(31,3)$ & $180(68,7)$ \\
\hline
\end{tabular}

À l'instar des sucres ajoutés, on pourrait réduire la teneur en sodium et augmenter la teneur en fibres dans les céréales pour déjeuner ainsi que dans d'autres aliments transformés au moyen de cibles fixées par le gouvernement fédéral, suivies de l'adoption d'un règlement obligeant le fabricant à apporter des modifications en cas de faible conformité. Il importe toutefois de garder à l'esprit que la modification de la composition d'un aliment peut être difficile, en particulier lorsque des nutriments comme le sodium, les gras et le sucre jouent souvent un rôle technique dans les produits ${ }^{43}$. Le sel, par exemple, est un agent de conservation qui empêche l'altération des aliments, tandis que le sucre est utilisé pour la texture ou la sensation en bouche, la conservation et comme agent de remplissage.

Malgré les défis que pose cette situation, l'industrie canadienne des aliments et des boissons a été en mesure, par le passé, de transformer la composition des produits de façon positive. Par exemple, une évaluation récente en Colombie-Britannique a montré que l'utilisation de gras trans dans les aliments servis au restaurant a connu une baisse marquée depuis le lancement d'une initiative en $2009^{44}$. Des données probantes révèlent également que les teneurs en sodium et en sucre dans les céréales à déjeuner varient d'un pays à l'autre, ce qui indique qu'une composition différente est possible : les céréales Fruit Loops de Kellogg's, par exemple, renferment $25 \mathrm{~g}$ de sucre par $100 \mathrm{~g}$ de produit au Koweït, tandis que le même produit en contient $40 \mathrm{~g}$ au Mexique et au Brésil ${ }^{45}$.

Outre un changement de composition des céréales pour déjeuner, il est recommandé d'adopter des règlements qui restreignent le marketing d'aliments et de boissons ciblant les enfants sur l'emballage des produits. Des travaux de recherche menés au Canada ont montré que l'autoréglementation du marketing par l'intermédiaire de l'Initiative canadienne pour la publicité sur 
les aliments et boissons destinés aux enfants s'est avérée inefficace dans la publicité télévisée et numérique : les enfants continuent à être énormément exposés au marketing des aliments malsains dans ces médias ${ }^{15-17}$. L'échec de l'autoréglementation a aussi été observé dans d'autres pays comme les États-Unis et l'Australie ${ }^{46,47}$. En particulier, étant donné que l'Initiative n'inclut pas l'emballage, les enfants sont bombardés d'éléments de marketing sur les boîtes de céréales pour déjeuner, attirantes pour eux. La Coalition Arrêtons la pub destinée aux enfants, qui réunit plus de 25 grands organismes non gouvernementaux des domaines de la santé et de l'enfance sous la direction de la Fondation des maladies du cœur et de l'AVC et de la Childhood Obesity Foundation, préconise une réglementation du marketing des aliments et des boissons ciblant les enfants et les jeunes au Canada. Dans les Principes d'Ottawa élaborés récemment, qui formulent des recommandations pour l'élaboration de politiques, la Coalition recommande de restreindre le marketing de tous les aliments et boissons ciblant les enfants de 16 ans et moins ${ }^{48}$. Il s'agit d'élargir la définition du marketing pour inclure l'emballage de produits aux formes de marketing s'adressant aux enfants. Cette requête a été récemment mentionnée dans la lettre de mandat du Premier ministre à la ministre de la Santé ${ }^{49}$. Santé Canada est également en train de travailler sur cette question, et des règlements gouvernementaux devraient être proposés à l'automne $2018^{50}$. À l'automne 2016, la sénatrice Greene Raine a en outre présenté au Sénat le projet de loi S-228 sur la publicité s'adressant aux enfants $^{51}$, qui demande qu'une modification soit apportée à la Loi sur les aliments et drogues afin d'interdire toute publicité d'aliments et de boissons malsains s'adressant aux enfants de moins de 17 ans, quel que soit le média, et incluant l'emballage de produits. La réglementation du marketing d'aliments ciblant les enfants nivellerait les conditions imposées aux fabricants d'aliments pour déjeuner et les empêcherait de faire de la publicité s'adressant aux enfants sur leur emballage, ce qui pourrait avoir un effet positif sur les efforts de prévention de l’obésité juvénile.

\section{Forces et limites}

Cette étude est la première à avoir examiné un vaste échantillon de céréales pour déjeuner canadiennes et à les avoir classé systématiquement en fonction de leur contenu nutritionnel et selon si leur emballage ciblait ou non les enfants. C'est aussi la première étude à évaluer les bienfaits pour la santé d'une large gamme de produits de fabricants de céréales pour déjeuner. L'utilisation d'un système de profilage nutritionnel validé (le modèle de profil nutritionnel du Royaume-Uni) pour classer les céréales comme "plus saines » ou « moins saines » constitue également un atout.

Parmi les points faibles, mentionnons que les céréales évaluées étaient celles d'un échantillon de commodité provenant de cinq grandes chaînes d'alimentation au Canada, et ce, même si on a tenté de choisir des commerces situés dans différentes régions d'Ottawa et de Gatineau afin d'obtenir un échantillon complet des céréales offertes sur le marché. Comme les céréales ont été analysées seulement à Ottawa (Ontario) et à Gatineau (Québec), les résultats ne sont pas généralisables aux céréales vendues dans d'autres régions du Canada. Néanmoins, les gammes de produits des grands fabricants de céréales sont relativement stables d'un bout à l'autre du pays. Les recherches à venir devraient examiner d'autres aliments destinés aux enfants pouvant avoir une faible valeur nutritionnelle, comme les aliments de restauration rapide, les friandises et les collations.

\section{Conclusion}

Les résultats de notre étude soulignent l'importance d'inclure l'emballage des produits dans les restrictions relatives au marketing des aliments et des boissons auprès des enfants, compte tenu des taux élevés d'obésité juvénile au Canada, des données probantes faisant ressortir le rôle et l'impact du marketing des aliments et des données probantes actuelles indiquant que les céréales pour déjeuner destinées aux enfants ne constituent pas des choix sains. En outre, il est essentiel que les entreprises alimentaires modifient la composition de leurs céréales pour déjeuner destinées aux enfants. Cet objectif est réalisable grâce à l'établissement de cibles par le gouvernement fédéral. En réduisant la quantité de sucres ajoutés et de sodium dans leurs produits et en augmentant la teneur en fibres, les entreprises canadiennes de céréales à déjeuner pourraient avoir une influence positive sur la santé des enfants canadiens.

\section{Remerciements}

Merci à Sony Subedi pour son aide lors de la collecte de données, à Arianne Kent pour son aide relative à la traduction et à la révision ainsi qu'à Elise Pauzé pour son aide concernant les références.

\section{Références}

1. Ogden CL, Carroll MD, Kit BK, Flegal KM. Prevalence of childhood and adult obesity in the United States, 2011-2012. JAMA. 2014;311(8):806-814.

2. World Health Organization. Obesity: Situation and trends [Internet]. Genève (CH): WHO [date inconnue; consulté le 6 décembre 2016]. En ligne à : http://www.who.int/gho/ncd/risk _factors/obesity_text/en/

3. Robert KC, Shields $M$, de Groh M, Aziz A, Gilbert JA. L'embonpoint et l'obésité chez les enfants et les adolescents : résultats de l'Enquête canadienne sur les mesures de la santé de 2009 à 2011. Rapports sur la santé. 2012;23(3):39-43.

4. Janssen, I. The public health burden of obesity in Canada. Can J Diabetes. 2013;37(2):90-96.

5. McGinnis JM, Gootman JA, Kraak VI, editors. Food marketing to children and youth: threat or opportunity? Washington (DC): The National Academies Press; 2006. 516 p.

6. Sadeghirad B, Duhaney T, Motaghipisheh $\mathrm{S}$, Campbell NR, Johnston BC. Influence of unhealthy food and beverage marketing on children's dietary intake and preference: a systematic review and meta-analysis of randomized trials. Obes Rev. 2016;17(10): 945-959.

7. Potvin Kent MP, Dubois L, Wanless A. A nutritional comparison of foods and beverages marketed to children in two advertising policy environments. Obesity (Silver Spring). 2012;20(9): 1829-1837.

8. Batada A, Seitz MD, Wootan MG, Story M. Nine out of 10 food advertisements shown during Saturday morning children's television programming are for foods high in fat, sodium, or added sugars, or low in nutrients. $\mathrm{J}$ Am Diet Assoc. 2008;108(4):673-678. 
9. Zuppa JA, Morton H, Mehta KP. Television food advertising: counterproductive to children's health? A content analysis using the Australian Guide to Healthy Eating. Nutr Diet. 2003;60(2):78-85.

10. World Health Organization (WHO). A framework for implementing the set of recommendations on the marketing of foods and non-alcoholic beverages to children. Genève (CH) : WHO; 2012. En ligne à : http://www.who.int / d i e t p hy s i c a la c t i vit y /MarketingFramework2012.pdf

11. Les normes canadiennes de la publicité. Initiative canadienne pour la publicité sur les aliments et les boissons destinée aux enfants [Internet]. Toronto (Ont.) : Les normes canadiennes de la publicité; 2016 [mise à jour]. En ligne à : http://www.adstandards.com/fr /initiativeenfants/CCFBAI_FR.pdf

12. Office de la protection du consommateur. Loi sur la protection du consommateur [Internet]. Québec (QC) : Office de la protection du consommateur; 2016 [mise à jour : avril 2017]. En ligne à : http://legisquebec.gouv.qc.ca /fr/pdf/cs/P-40.1.pdf

13. Frazier III WC, Harris JL. Trends in television food advertising to young people: mise à jour de 2015 [Internet]. Hartford (CT): UConn Rudd Center for Food Policy and Obesity; 2016. En ligne à : http://uconnruddcenter.org /files/TVAdTrends2016.pdf

14. Federal Trade Commission. A review of food marketing to children and adolescents: follow-up report [Internet]. Washington (DC): Federal State Commission; 2012. En ligne à : https:// www.ftc.gov/sites/default/files /documents/reports/review-food -marketing-children-and-adolescentsfollow-report/121221 foodmarketingrep ort.pdf

15. Potvin Kent M, Wanless A. The influence of the Children's Food and Beverage Advertising Initiative: change in children's exposure to food advertising on television in Canada between 2006-2009. Int J Obes (Lond). 2014; 38(4):558-562.
16. Potvin Kent M, Dubois L, Wanless A. Food marketing on children's television in two different policy environments. Int J Pediatr Obes. 2011;6(Suppl 3):e433-e441.

17. Fondation des maladies du cœur et de l'AVC. Nos enfants sont bombardés. Comment le marketing de l'industrie des aliments et des boissons met en péril la santé de nos enfants et de nos jeunes. Bulletin de santé de 2017 [Internet]. Fondation des maladies du cœur et de l'AVC; 2017. En ligne à : https://www.coeuretavc.ca/-/media /pdf-files/iavc/2017-heart-month /coeuretavc-bulletinsante2017fr.ashx

18. Harris JL, Schwartz MB, Brownell KD, et al. Cereal FACTS: Limited progress in the nutrition quality and marketing of children's cereals [Internet]. New Haven (CT): Rudd Center for Policy and Obesity; 2012. En ligne à : http:// www.cerealfacts.org/media/Cereal _FACTS_Report_2012_7.12.pdf

19. Schwartz MB, Vartanian LR, Wharton $\mathrm{CM}$, Brownell KD. Examining the nutritional quality of breakfast cereals marketed to children. J Am Diet Assoc. 2008;108(4):702-705.

20. Elliott C. Assessing 'fun foods': nutritional content and analysis of supermarket foods targeted at children. Obes Rev. 2008;9(4):368-377.

21. Te Morenga L, Mallard S, Mann J. Dietary sugars and body weight: systematic review and meta-analyses of randomised controlled trials and cohort studies. BMJ [Internet]. 2013; 346:e7492. En ligne à : http://dx.doi .org/10.1136/bmj.e7492

22. Santé Canada. Règlement modifiant le Règlement sur les aliments et drogues (étiquetage nutritionnel, autres disposition d'étiquetage et colorants alimentaires) [Internet]. Ottawa (Ont.) : Santé Canada; 2016. En ligne à : http://www.gazette.gc.ca/rp-pr/p2 /2016/2016-12-14/html/sor-dors305 -fra.php

23. Sturgeon J. Here's who's really winning Canada's grocery wars [Internet] Global News, 2014 [mise à jour le 19 novembre 2014]. En ligne à : http:// globalnews.ca/news/1678970/heres -whos-really-winning-canadas-grocery -wars/
24. Mensink RP, Katan MB. Effect of dietary trans fatty acids on high-density and low-density lipoprotein cholesterol levels in healthy subjects. $\mathrm{N}$ Engl J Med. 1990;323(7):439-445.

25. Agence canadienne d'inspection des aliments (ACIA). Liste d'ingrédients et allergène : Déclaration [Internet]. Ottawa (Ont.) : ACIA; [mise à jour : juillet 2016]. En ligne à : http://www inspection.gc.ca/aliments/etiquetage /1-etiquetage-des-aliments-pour-1 -industrie/liste-d-ingredients-et-allergene /fra/1383612857522/1383612932341 ?chap $=2$

26. UK Department of Health. Nutrient Profiling Technical Guidance January 2011 [Internet]. London (UK): Department of Health; 2011. En ligne à : https://www.gov.uk/government /uploads/system/uploads/attachment _data/file/216094/dh_123492.pdf

27. Arambepola C, Scarborough P, Rayner M. Validating a nutrient profile model. Public Health Nutr. 2008;11(4):371-378.

28. Scarborough P, Boxer A, Rayner M, Stockley L. Testing nutrient profile models using data from a survey of nutrition professionals. Public Health Nutr. 2007;10(4):337-345.

29. Romero-Fernández MM, RoyoBordonada MA, Rodríguez-Artalejo F. Evaluation of food and beverage television advertising during children's viewing time in Spain using the UK nutrient profile model. Public Health Nutr. 2013;16(7):1314-1320.

30. Potvin Kent M, Martin CL, Kent EA. Changes in the volume, power and nutritional quality of foods marketed to children on television in Canada. Obesity (Silver Spring). 2014;22(9): 2053-2060.

31. Bernstein JT, Schermel A, Mills CM, L'Abbé MR. Total and free sugar content of Canadian prepackaged foods and beverages. Nutrients. 2016;8(9): 582-604.

32. World Health Organization (WHO). Guideline: sugars intake for adults and children [Internet]. Genève $(\mathrm{CH})$ : WHO; 2015. En ligne à : http://apps.who.int /iris/bitstream/10665/149782/http:// apps.who.int//iris/bitstream/10665 /149782/1/9789241549028_eng.pdf ?ua $=1$ 
33. Santé Canada. Besoins énergétiques estimatifs [Internet]. Ottawa (Ont.) : Santé Canada [mise à jour : novembre 2011]. En ligne à : https://www .canada.ca/fr/sante-canada/services /aliments-nutrition/guide-alimentaire -canadien/renseignements-base-guide -alimentaire/besoins-energetiques -estimatifs.html

34. Hu FB. Resolved: there is sufficient scientific evidence that decreasing sugar-sweetened beverage consumption will reduce the prevalence of obesity and obesity-related diseases. Obes Rev. 2013;14(8):606-619.

35. HM Government (UK). Childhood obesity: a plan for action [Internet]. London (UK): HM Government [mise à jour : janvier 2017]. En ligne à : https://www.gov.uk/government /publications/childhood-obesity-a -plan-for-action

36. Yang Q, Zhang Z, Kuklina EV, et al. Sodium intake and blood pressure among US children and adolescents. Pediatrics. 2012;130(4):611-619.

37. He FJ, MacGregor GA. Importance of salt in determining blood pressure in children: meta-analysis of controlled trials. Hypertension. 2006;48(5): 861-869.

38. World Health Organization (WHO). Guideline: sodium intake for adults and children [Internet]. Genève $(\mathrm{CH})$ : WHO; 2012. En ligne à : http://apps .who.int/iris/bitstream/10665/77985 /1/9789241504836_eng.pdf?ua = 1\&ua $=1$

39. Institute of Medicine. Chapter 7: Dietary, functional and total fibre. In: Dietary reference intakes for energy, carbohydrate, fiber, fat, fatty acids, cholesterol, protein, and amino acids. Washington (DC): The National Academies Press; 2005. p. 339-422.

40. Kranz S, Brauchla M, Slavin JL, Miller KB. What do we know about dietary fiber intake in children and health? The effects of fiber intake on constipation, obesity, and diabetes in children. Adv Nutr. 2012;3(1):47-53.

41. Kimm SY. The role of dietary fiber in the development and treatment of childhood obesity. Pediatrics. 1995;96(5): 1010-1014.
42. Nicklas TA, Farris RP, Myers L, Berenson GS. Dietary fiber intake of children and young adults: the Bogalusa Heart Study. J Am Diet Assoc. 1995;95(2):209-214.

43. Buttriss JL. Food reformulation: the challenges to the food industry. Proc Nutr Soc. 2013;72:61-69.

44. Heart and Stroke Foundation and Government of British Columbia. BC trans fat initiative score card: update April 2014 [Internet]. Victoria (BC): HSF and Government of British Columbia; 2014. En ligne à : http://www.restricttransfat .ca/media/upload/file/trans-fat -initiative-score-card-april-2014.pdf

45. World Action on Salt and Health (WASH). Global breakfast cereals survey 2016 - full data [Internet]. WASH; 2016. En ligne à : http://www .worldactiononsalt.com/less/surveys /2016/190144.pdf

46. Harris J, Schwartz MB, Shehan C, Hyary M, Appel J, Haraghey et al. Snack FACTS 2015: evaluating snack food nutrition and marketing to youth [Internet]. Hartford (CT): Rudd Center for Food Policy and Obesity; 2015. En ligne à : http://www.uconnruddcenter .org/files/Pdfs/SnackFACTS_2015 _Fulldraft03.pdf

47. Smithers LG, Lynch J W, Merlin T. Industry self-regulation and TV advertising of foods to Australian children. J Paediatr Child Health. 2014;50(5): 386-392.

48. Coalition Arrêtons la pub destinée aux enfants. Les Principes d'Ottawa [Internet]. Fondation des maladies du cœur et de l'AVC; 2016. En ligne à : http://stopmarketingtokids.ca/fr/nos -recommendations-de-politiques -publiques/

49. Trudeau J. Lettre de mandat de la ministre de la Santé [Internet]. Ottawa (Ont.) : Cabinet du Premier ministre; 2015. En ligne à : http://pm.gc.ca/fra /lettre-de-mandat-de-la-ministre-de -la-sante

50. Santé Canada. Vers la restriction de la publicité de boissons et d'aliments mauvais pour la santé destinée aux enfants: document de travail aux fins de la consultation publique [Internet]. Santé Canada; 2017. En ligne à : https:// www.consultationssainealimentation .ca/document-de-travail
51. Sénat du Canada. Projet de loi S-228 : Loi modifiant la Loi sur les aliments et drogues (interdiction de faire de la publicité d'aliments et de boissons s'adressant aux enfants) [Internet]. Ottawa (Ont.) : Sénat du Canada (Première session, quarante-deuxième législature); 2016. En ligne à : http:// www.parl.ca/DocumentViewer/fr /42-1/projet-loi/S-228/premiere -lecture 